\title{
Interpenetrating Polymer Network Hydrogels Based on Gelatin and PVA by Biocompatible Approaches: Synthesis and Characterization
}

\author{
Eltjani-Eltahir $\mathrm{Hago}^{1}$ and Xinsong $\mathrm{Li}^{2}$ \\ ${ }^{1}$ Department of Chemistry and Chemical Engineering, Southeast University, Nanjing 210096, China \\ ${ }^{2}$ School of Chemistry and Chemical Engineering, Southeast University, Nanjing 211189, China \\ Correspondence should be addressed to Eltjani-Eltahir Hago; eltjanihago@gmail.com
}

Received 14 March 2013; Revised 29 May 2013; Accepted 31 May 2013

Academic Editor: Delia Brauer

Copyright (C) 2013 E.-E. Hago and X. Li. This is an open access article distributed under the Creative Commons Attribution License, which permits unrestricted use, distribution, and reproduction in any medium, provided the original work is properly cited.

In this work, a new approach was introduced to prepare interpenetrating polymer network PVA/GE hydrogels by cross-linking of various concentration gelatin in the presence of transglutaminase enzyme by using the freezing-thawing cycles technique. The effects of freezing-thawing cycles on the properties of morphological characterization, gel fraction, swelling, mechanical, and MTT assay were investigated. The IPN PVA/GE hydrogels showed excellent physical and mechanical Properties. MTT assay data and the fibroblasts culture also showed excellent biocompatibility and good proliferation. This indicates that the IPN hydrogels are stable enough for various biomedical applications.

\section{Introduction}

Polyvinyl alcohol (PVA) hydrogels are a gel polymer known for the quality of biocompatibility that have been used in many biomedical applications, for example, as implants [1], artificial devices [2], contact lenses [3], drug delivery devices [4], and also wound dressings [5-7]. The following methods were used on a large scale: chemical cross-linking [8]; glutaraldehyde as the cross-linking agent [9]; cross-linking through $\gamma$ radiation [10, 11], ultraviolet radiation [12], and by using cycles of freezing-thawing of successive $[13,14]$. The last one is very convenient and biocompatible, while the molecular bonds (hydrogen bonds), which form through the freezingthawing process of aqueous solutions of PVA, act as an efficient crosslinks [15].

The extent of poly(vinyl alcohol) (PVA) hydrogels for various biomedical applications has been realized because of their excellent biocompatibility and chemical stability. The freezing-thawing techniques used in the preparation of the PVA hydrogels showed good mechanical strength and have been examined for use as intervertebrate disk intended [16], artificial meniscus [17], and a contact lens [18, 19]. However, manufactured polymers, due to the lack of bioactive moieties, have no biological activity compared with natural polymers. The concept of integrating artificial materials with cell locations approved sites of naturally derived materials is very attractive. To achieve this result, efforts were made to incorporate cell adhesion of synthetic biomaterials [20].

Gelatin (GE) is a protein produced by partial denaturalization of collagen extracted from the bones, connective tissues, organs, and some intestines of animals such as domesticated cattle, porcine, and horses. Gelatin presents biological activities because of the natural origin, which makes it suitable for use of an ingredient of wound dressing, scaffolds for tissue engineering, and drug delivery carriers. Gelatin hydrogels are common for the applications in medical areas [21]. Hydrogels are insoluble hydrophilic polymers having a highwater content and tissue like mechanical properties that make them highly attractive scaffolds for implantation in empty tubular nerve prosthesis or for direct injection at the lesion site to enhance cell attachment and growth. Gelatin hydrogels have often crossed-linked by chemical approach using crosslinks, such as glutaraldehyde, to improve elasticity, consistency, and stability. However, the chemical cross-linker may lead to toxic effect on physiological environment, due to the presence of residual crosslinks. Therefore, it is highly 
demanded to develop biocompatible mild cross-linking methods to prepare hydrogels [20].

Transglutaminases are widely distributed in various organisms, including vertebrates, invertebrates, plants, and microorganism, and are reportedly responsible for certain biological events such as epidermal keratinization, blood coagulation, and regulation of erythrocyte membranes. A microbial transglutaminase isolated from the culture medium of Streptoverticillium mobaraense has become commercially available [22]. Unlike TGases from many sources, the mTG possesses many features, including $\mathrm{Ca}^{2+}$ independence, a broader substrate specificity for acyl donors, a smaller molecule size, and a higher reaction rate, which makes them suitable for industrial applications. Currently, this mTG has been successfully applied in the food industry for improving the physical properties and texture of protein-related foods [23]. More recently, the use of mTG to modify gelatin has also been reported [24, 25]. The enzymatic cross-linking is a biocompatible approach to enhance the mechanical properties of gelatin hydrogels. An interpenetrating polymer network (IPN) is a composite of polymers, exhibiting varied characteristics, which is obtained when one polymer network is synthesized or cross-linked independently in the instantaneous presence on the other [26]. The IPN is a combination of at least two polymer chains each in network form, which is synthesized and/or cross-linked in the immediate presence on the other without any covalent bonds between them [27] or the two or more networks can be envisioned to be entangled in such a way that they are concatenated and cannot be pulled apart but not bonded to each other by any chemical bond [28]. Many researchers have been focusing on the development of polymer blends with IPN structure. Recently, hydrogels with IPN structure have attracted much attention because of their potential application in the biomedical area. Kurokawa et al. invented double network hydrogels with superior toughness [29].

In this paper, a new approach was introduced to prepare interpenetrating polymer network PVA/GE hydrogels by a combination of enzymatic and physical methods, used freezing-thawing process, and in situ with synthesis of gelatin/ mTG in PVA solution. The influence on the contents and dispersed condition of gelatin in PVA matrixes on the hydrogels mechanical strength was investigated in order to obtain applicable hydrogels. The morphology and crystalline structures of interpenetrating polymer network PVA/GE were also observed by some experimental analysis techniques, such as scanning electronic microscope (SEM). Moreover, in order to understand the initial behavior of fibroblasts cells, proliferation was assessed in vitro using fibroblast like L 929 cell culture.

\section{Experimental}

2.1. Materials. PVA with a degree of polymerization of $1750 \pm$ 50 and hydrolysis degree values of greater than $99 \%$ was purchased from Beijing Organic Chemical Plant, China. Gelatin (GE) (type A, 300 bloom from porcine) was obtained
TABLE 1: Composition of IPN PVA/GE hydrogels.

\begin{tabular}{lcccc}
\hline Hydrogels & IPN-1 & IPN-2 & IPN-3 & IPN-4 \\
\hline Gelatin \%w/v & 0 & 2 & 5 & 7 \\
PVA \%w/v & 15 & 15 & 15 & 15 \\
\hline
\end{tabular}

The concentration of $\mathrm{mTG}$ at a range of $5-20 \mathrm{U} / \mathrm{mL}$.

(Sigma-Aldrich, St. Louis, MO, USA). The source of transglutaminase was the commercial product obtained from Yiming Biological Products Co., Ltd. (Jiangsu, China). As determined by a colorimetric hydroxamate method [30], the enzyme activity of mTG was 102 (U/g) of powder. All other regents used in the paper were of analytical grade.

2.2. Moisture Determination of Gelatin. In order to know the accurate concentration of the gelatin solution prepared subsequently, it is necessary to know the moisture content of the gelatin. The moisture of gelatin was determined according to Chinese standards GB/T 5009.3-2003 [31].

2.3. Preparation of Gelatin and $m T G$ Blends. A concentrated gelatin solution was prepared by adding $50 \mathrm{gm}$ of gelatin to $100 \mathrm{~mL}$ of warm deionized water and mixing at $50^{\circ} \mathrm{C}$ until the protein was dissolved. Aliquots from this concentrated gelatin solution were then mixed with deionized water to prepare solutions with differing gelatin concentrations $(0 \%, 2 \%$, $5 \%$, and $7 \% \mathrm{w} / \mathrm{v})$. The gelatin solutions were stored at $4^{\circ} \mathrm{C}$. A concentrated enzyme solution was prepared for each experiment by mixing $0.5 \mathrm{gm}$ of $\mathrm{mTG}$ per $5 \mathrm{~mL}$ of deionized water at room temperature. This enzyme solution was stored at $4^{\circ} \mathrm{C}$ until it was ready for use.

\subsection{Preparation of IPN PVA/Gelatin Hydrogels. IPN PVA/GE} hydrogels were prepared by cyclic freezing-thawing method. For this purpose, aqueous solutions containing $15 \%$ by weight PVA with different amounts of gelatin, (i.e., $0 \%, 2 \%, 5 \%$, and $7 \%)$ by weight, were used. The $(15 \% \mathrm{w} / \mathrm{v})$ of PVA solution was prepared at $95^{\circ} \mathrm{C}$. A varying amount of solution's gelatin and $\mathrm{mTG}$ was mixed slowly to the PVA solution at room temperature to induce cross-linking. The mixture was cast between glass slides with $3 \mathrm{~mm}$ thick spacers then incubated at $45^{\circ} \mathrm{C}$ for $4 \mathrm{~h}$ to achieve complete cross-linking. Then, they were physically cross-linked by three freeze-thaw cycles, which consisted of freezing at $-20^{\circ} \mathrm{C}$ for $24 \mathrm{~h}$ and thawing at room temperature $21^{\circ} \mathrm{C}$ for $24 \mathrm{~h}$, respectively, to produce IPN hydrogels. The IPN hydrogels films had a thickness of $3 \mathrm{~mm}$. Table 1 shows the composition of IPN PVA/GE hydrogels.

2.5. Morphological Characterization. For morphological characterization, hydrogels after swelling (equilibrium) in water were freeze-dried using a freeze drier (Christ, Germany, Alpha 1-2) at $-52^{\circ} \mathrm{C}$ for $12 \mathrm{~h}$. Transverse sections were cut from freeze-dried film samples using a cold knife. Samples were then examined by a scanning electron microscope (SEM JSM-6360LV, a voltage of $20 \mathrm{KV}$, China). The working face of the samples was sprayed with gold in advance. The observed morphologies for each SEM fractograph were analyzed using 
Sigma Scan Pro software. Quantitative analysis of the pore size was obtained from structural indices measured from scaffold samples. The density of the solid material (film) was calculated according to the mass fractions $\left(M_{x}\right.$ where $x$ refers to the constituent material) and densities of the constituent materials $\left(\rho_{x}\right)$, by assuming that the total mass and volume remain the same before and after reaction [32], using (1)

$$
\rho_{s}=\left[\frac{\left(M_{\mathrm{PVA}}+M_{\mathrm{GE}}+M_{\mathrm{mTG}}\right)}{\left[\left(M_{\mathrm{PVA}} / \rho_{\mathrm{PVA}}\right)+\left(M_{\mathrm{GE}} / \rho_{\mathrm{GE}}\right)+\left(M_{\mathrm{mTG}} / \rho_{\mathrm{mTG}}\right)\right]}\right] .
$$

2.6. Gel Fraction. The weight ratio of the dried hydrangeas in rinsed and unrinsed conditions or gel fraction can be assumed as an index of the degree of cross-linking. Therefore, the gel fraction of samples can be calculated as follows (2):

$$
\text { Gel fraction }(\%)=\left(\frac{W_{f}}{W_{i}}\right) \times 100,
$$

where $W_{f}$ and $W_{i}$ are the weights of the dried hydrogel after and before rinsing and extraction. To perform gel fraction measurement, preweighed slice of each sample was dried under vacuum at room temperature until observing no change in its mass. Nearly identical weight of another slice of the same sample was immersed into excess of distilled water for 4 days to rinse away amorphous or soluble part. Subsequently, the immersed sample was removed from distilled water and dried at room temperature under vacuum until the dried mass showed constant weight.

\subsection{Equilibrium Degree of Swelling and Equilibrium Water} Content. For swelling experiments, the vacuum-dried films were immersed in excess distilled water at room temperature $\left(\sim 30^{\circ} \mathrm{C}\right)$ until reaching to an equilibrium state. Then, the swelled samples were withdrawn from distilled water and weighed after gentle surface wiping using absorbent paper. The equilibrium degree of swelling (EDS) and equilibrium water contents (EWC) were calculated, respectively, as follows [33]:

$$
\begin{aligned}
& \operatorname{EDS}(\%)=\left[\frac{\left(W_{s}-W_{d}\right)}{W_{d}}\right] \times 100, \\
& \operatorname{EWC}(\%)=\left[\frac{\left(W_{s}-W_{d}\right)}{W_{s}}\right] \times 100,
\end{aligned}
$$

where $W_{s}$ is the swollen weight of the sample of the equilibrium state and $W_{d}$ is the final dry weight of the extracted sample.

2.8. Mechanical Properties. The samples were cut into a slice shape with a thickness of $3 \mathrm{~mm}$ and their mechanical properties, including tensile strength at break, were determined. The mechanical properties were measured at a temperature of $17^{\circ} \mathrm{C}$ and humidity of $60 \%$, at a crosshead speed of 50.000 ( $\mathrm{mm} / \mathrm{min}$ ) using Instron tester-4466, type: 42/43/4400. The elastic moduli of the IPN PVA/GE hydrogels were determined by performing constant strain-rate compression measurements on an Instron 4466 mechanical tester at room temperature. The hydrogel sample, $15 \mathrm{~mm}$ in diameter and $10 \mathrm{~mm}$ in height, was tested at a rate of 5.000 (pts/secs) and humidity of $40 \%$, at a crosshead speed of $1.5000 \mathrm{~mm} / \mathrm{min}$. The results are expressed as mean value \pm standard deviation with the confidence level of $95 \%$.

2.9. Cell Viability Assay. Cellular survivability evaluation against cross-linked PVA, GE, and IPN PVA/GE blend has been evaluated by MTT (3-[4,[5-dimethylthiazol-2-yl]-2,5diphenyl tetrazolium bromide) assay [34]. This standard test method is based on exposing L929 cells to the fluid extract of the test materials and control materials. The extract solution of the PVA, GE, or cross-linked IPN PVA/GE blend was prepared according to the protocol as outlined in ISO 10993 [35]. In brief, to determine the cytotoxicity of osteoblast-like L929 cells on the IPN hydrogels samples, samples were incubated with DMEM media at $37^{\circ} \mathrm{C}$ for $72 \mathrm{~h}$ in an incubator. The extract solutions were diluted serially with the media $(0 \%, 12.5 \%, 25 \%, 50 \%$, and $100 \%)$. For the control, $100 \%$ media were used to compare among the dilution, and then, $200 \mathrm{~mL}$ of extracting and diluted solution was added in a 96-well plate. The 96-well plate was previously $(24 \mathrm{~h}$ before adding the extract solutions) incubated and coated with fibroblasts cells $\left(1 \times 10^{4}\right.$ cells/well $)$. The plate was then incubated in a $\mathrm{CO}_{2}$ incubator at $37^{\circ} \mathrm{C}$ for 1 day, 3 days, and 5 days. In MTT, assay cell survivability is measured through the production of purple colors by the reaction of dehydrogenase enzymes of living cells and MTT. Metabolically active and viable cells produce mitochondrial dehydrogenase enzymes during incubation in media, which can be read out through the change of color intensity by a spectrophotometer. The optical density (OD) corresponds to the viable cell numbers. Therefore, cell survival and proliferation at the different dilutions and time points were quantified by adding $100 \mu \mathrm{L}$ of the MTT solution $(20 \mu \mathrm{g} / 100 \mathrm{~mL})$ to each of the wells. After $4 \mathrm{~h}$ of incubation, the OD values of the solution were measured using an BIORAD reader (Model 680, Microplate reader) at a wavelength of $595 \mathrm{~nm}$.

2.10. Optical Microscope Study. In order to observe directly whether the viable cell numbers increases after treating the osteoblast like L929 cells with sample extracts after three freezing-thawing cycles and day 5, an inverted light microscope (Olympus, $1 \times 71$ ) attached with LCD monitor was used. After 70-80\% confluent growth in the subculture flask, cells were trypsinized ( $0.25 \%$ Trypsin-EDTA), detached, and counted. Approximately, $1 \times 10^{3}$ cells $/ \mathrm{mL}$ media was pipette into the wells of a Microtiter plate containing 24 wells. The plate was then incubated in a $\mathrm{CO}_{2}$ incubator $\left(5 \% \mathrm{CO}_{2}, 37^{\circ} \mathrm{C}\right)$ for $24 \mathrm{~h}$. after seeding cells into the wells of Microtiter plates, media were removed carefully and replaced with the extract solution of the samples, and the Microtiter plate was kept back into the $\mathrm{CO}_{2}$ incubator either for 5 days. After finishing each of the incubation periods, cell growth in the Microtiter plate was observed using an inverted light microscope.

2.11. Statistical Analysis. All experiments were done in triplicate, and the results were expressed as mean $\pm \mathrm{SD}$, and the means were analyzed by one-way ANOVA at a $P$ value of 0.05 . 


\section{Results and Discussion}

3.1. Moisture of Gelatin. The result of moisture in gelatin used in the study is $11.51 \%$. As the gelatin solutions were prepared, the moisture content of the gelatin was taken into account.

3.2. Morphological Characterization. In applied freezingthawing process, the structure of the PVA and PVA/GE IPN hydrogels was determined by SEM. The densities of the crosslinked IPN hydrogels containing gelatin contents from $0 \mathrm{wt} . \%$ to $7 \mathrm{wt} . \%$ were investigated by density measurement, SEM. Tables 2 and 3 show the densities of IPN PVA/gelatin-mTG films $\left(\rho_{s}\right)$ calculated by (1). The density of the IPN PVA/ gelatin-mTG hydrogels was found to increase monotonously with increasing the gelatin content, namely, from $1.45 \mathrm{Mg} \mathrm{m}^{-3}$ to $1.49 \mathrm{Mg} \mathrm{m}^{-3}$. Figure 1 shows the scanning electron micrographs of transversal sections of the films after 1 (Figures 1(a1), 1(a2), 1(a3), and 1(a4))) and 3 (Figures 1(b1), 1(b2), 1(b3), and 1(b4)) freezing-thawing cycles. These micrographs show that increasing the concentration of gelatin blends and number of freezing-thawing cycles results in higher network arrangement, which leads to porous structure on the surface of IPN hydrogels. The internal porosity of samples shows significant correlation between number of freezing-thawing cycles, the chemical structure of additive and the pore size, and arrangement. Before adding the gelatin/mTG blends as modifying agent to PVA blend, the PVA hydrogel produced smooth structured films and exhibits a few irregular pores, and the morphology is not significantly changed as compared to the IPN PVA hydrogel Figures 1(a1) and 1(b1). As illustrated in Figures 1(b2), 1(b3), and 1(b4), IPN hydrogels after 3 freezing-thawing cycles shows highly irregular porous structure with the large pores size in the range of 50-100 $\mu \mathrm{m}$. Figures 1(a1) and 1(b1) after 1 and 3 cycles showed a fewer and smaller pore size in the range of $5-10 \mu \mathrm{m}$, as a consequence of the preparation technique. In the freeze-thaw process, pore size is in fact controlled by the size of the ice crystals, which could be adjusted by varying the rate of freezing. In Figures 1(a2), 1(a3), and 1(a4), IPN hydrogels after 1 cycle showed lower and smaller irregular porous size structure in the range of 20-40 $\mu \mathrm{m}$ than those obtained with Figures 1(b2), 1(b3), and 1 (b4). More significant change in sample morphology is observed after introducing the gelatin/mTG and increased the cycles as cross-linking agent. Therefore, it might be an optimum candidate for biomedical applications. In the films produced with PVA/GE mixtures, the presence of cracks and empty spaces increased with an increasing proportion of gelatin in the mixture.

3.3. Gel Fraction. Enzymatic cross-linking of gelatin and cyclic freezing-thawing of pure PVA leads to the formation of insoluble and entangled polymeric network in the IPN PVA/GE hydrogels. A typical dependency of the gel fractions to the quantity of gelatin incorporated into hydrogels is given in Tables 2 and 3 using (2). As seen, the gel fraction of samples is increased by increasing the number of freezing-thawing cycles and amounts of gelatin up to 7 (\%) by weight after (1 and 3) cycles. For example, the gel fraction of IPN hydrogel increases to 74 (\%) by adding $7 \%$ weight of gelatin after three cycles compared with the other IPN hydrogels after one cycle. On the other hand, Tables 2 and 3 exhibit a semilinear relationship between gel fraction, number of freezing-thawing cycles, and gelatin weight fraction in mixture hydrogels. The increase of the gel fraction may be attributed to the enzymatic cross-linked gelatin and the additional interactions between PVA and gelatin; besides, the bonds existed between PVA long neighboring chains induced crystallization, which caused an increase in gel fraction.

3.4. Degrees of Swelling and Water Content. In this work, the equilibrium degree of swelling (EDS) and equilibrium water content (EWC), as important swelling characteristics of hydrogels, were calculated using (3). These characteristics indicated the ability of absorption of fluids and exudates. Tables 2 and 3 demonstrate EDS and EWC of IPN PVA/GE hydrogels as a function of the amount of gelatin and the number of freezing-thawing cycles. Both parameters showed nearly similar decreasing trends by increasing the quantity of the gelatin content and the number of freezing-thawing cycles. The samples treated for three freeze-thaw cycles showed a much more swollen structure than others treated with one freeze-thaw cycle. The highly swollen hydrogel represents a high tight structure and a higher degree of crosslinking when compared with the hydrogels with lower swelling ratio. The comparison of the EDS and EWC data for the gel fraction values indicates that there is a logic relationship between these swelling characteristics and gel fraction, that is, more gel fractions lead to less EDS or EWC. Although the swelling characteristics of IPN PVA/GE hydrogels decrease due to presence of gelatin in comparison with pure PVA hydrogel, it seems that there have been high enough swelling capacity to be used as a suitable dressing even for healing exudative wounds.

3.5. Mechanical Properties. As mentioned before, the main interest in the production of IPN PVA/gelatin hydrogels is to achieve the materials with better mechanical properties. The investigated mechanical properties, that is, tensile strength, as well as strain at break were found to depend on the amount of gelatin and number of freezing-thawing cycles. As expected, cross-linking led to an improvement in the tensile strength. Based on these analyses, the composition of IPN hydrogels and three freezing-thawing cycles was considered the optimum conditions for the preparation of IPN hydrogels. The irregular arrangement within PVA hydrogel modified with gelatin/mTG after 3 freezing-thawing process (Table 3 ) has strong influence on mechanical properties. Independent of the enzymatic modification, the values for tensile strength of the films produced from IPN PVA/GE mixtures was significantly different varied as a function of the gelatin concentrations and number of freezing-thawing cycles in the mixture Tables 2 and 3. Table 3 shows that the highest values of compressive stress and tensile strength increases with increasing the concentration of gelatin and number of freezing-thawing cycles. It is observed that the tensile strength for pure PVA is $(0.41 \pm 0.07 \mathrm{MPa})$ and the elastic modulus is $(0.45 \pm 0.60 \mathrm{MPa})$. By addition of $2 \% \mathrm{w} / \mathrm{v}$ gelatin into PVA blend, the tensile strength increases to $(0.66 \pm 0.12 \mathrm{MPa})$ 
TABLE 2: The properties of IPN PVA/GE hydrogels after one freezing-thawing cycle.

\begin{tabular}{lcccc}
\hline Samples & IPN-1 & IPN-2 & IPN-3 & IPN-4 \\
\hline Gel fraction (\%) & $25 \pm 1.20$ & $36 \pm 1.35$ & $49 \pm 1.50$ & $58 \pm 1.65$ \\
EDS (\%) & $135 \pm 4.50$ & $132 \pm 3.75$ & $130 \pm 3.45$ & $42.5 \pm 2.50$ \\
EWC (\%) & $45 \pm 2.00$ & $44.5 \pm 2.25$ & $0.25 \pm 0.24$ & $42 \pm 2.75$ \\
Stress (MPa) & $0.11 \pm 0.14$ & $0.15 \pm 0.18$ & $34.56 \pm 12.90$ & $0.28 \pm 0.22$ \\
Strain (\%) & $18.23 \pm 4.60$ & $25.67 \pm 7.80$ & $0.56 \pm 0.62$ & $47.78 \pm 14.65$ \\
Elastic modulus $(\mathrm{MPa})$ & $0.35 \pm 0.45$ & $0.41 \pm 0.49$ & $0.52 \pm 0.15$ & $0.65 \pm 0.64$ \\
Tensile strength $(\mathrm{MPa})$ & $0.30 \pm 0.05$ & $0.42 \pm 0.09$ & 1.29 & $0.58 \pm 0.19$ \\
Solid density $\left(\rho_{s}\right)\left(\mathrm{Mg} \mathrm{m}^{-3}\right)$ & 1.13 & 1.20 & & 1.34 \\
\hline
\end{tabular}

TABLE 3: The properties of IPN PVA/GE hydrogels after three freezing-thawing cycles.

\begin{tabular}{|c|c|c|c|c|}
\hline Samples & IPN-1 & IPN-2 & IPN-3 & IPN-4 \\
\hline Gel fraction (\%) & $44 \pm 1.30$ & $58 \pm 1.60$ & $67 \pm 1.70$ & $74 \pm 1.90$ \\
\hline EDS (\%) & $165 \pm 3.50$ & $158 \pm 3.10$ & $149 \pm 2.80$ & $142 \pm 2.40$ \\
\hline EWC (\%) & $62 \pm 1.98$ & $61 \pm 1.92$ & $60 \pm 1.87$ & $59 \pm 1.82$ \\
\hline Stress $(\mathrm{MPa})$ & $0.25 \pm 0.12$ & $0.43 \pm 0.20$ & $0.64 \pm 0.34$ & $0.67 \pm 0.38$ \\
\hline Strain $(\%)$ & $25.50 \pm 6.40$ & $31.85 \pm 10.50$ & $48.64 \pm 14.00$ & $68.70 \pm 17.50$ \\
\hline Elastic modulus (MPa) & $0.45 \pm 0.60$ & $0.45 \pm 0.60$ & $0.87 \pm 1.75$ & $0.98 \pm 2.45$ \\
\hline Tensile strength (MPa) & $0.41 \pm 0.07$ & $0.66 \pm 0.12$ & $0.74 \pm 0.16$ & $0.75 \pm 0.18$ \\
\hline Solid density $\left(\rho_{s}\right)\left(\mathrm{Mg} \mathrm{m}^{-3}\right)$ & 1.27 & 1.45 & 1.47 & 1.49 \\
\hline
\end{tabular}

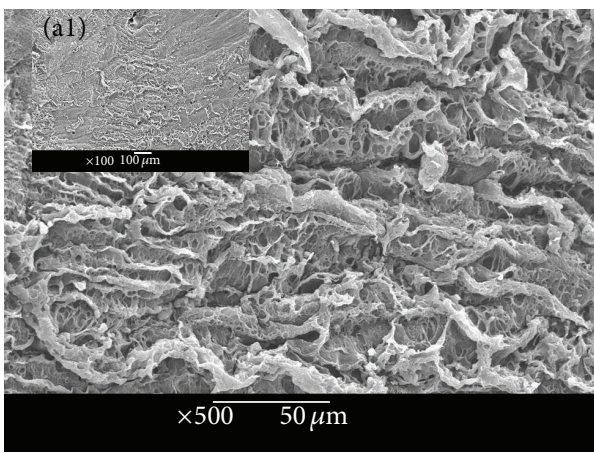

(b1)

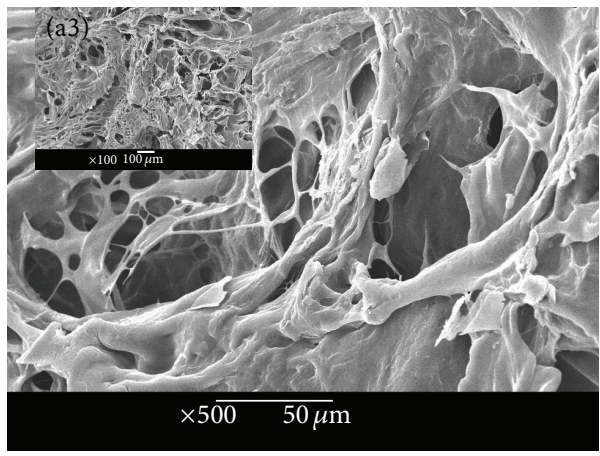

(b3)

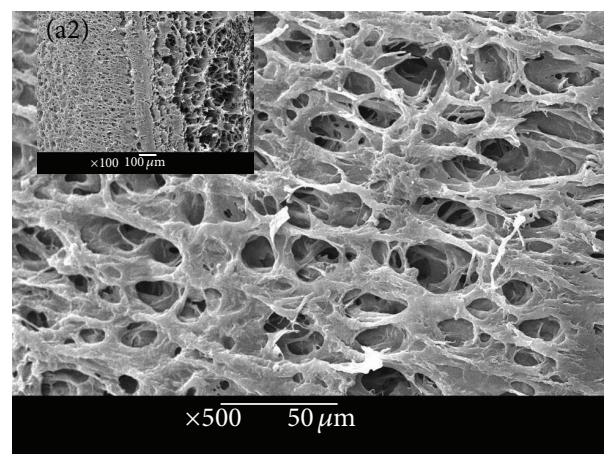

(b2)

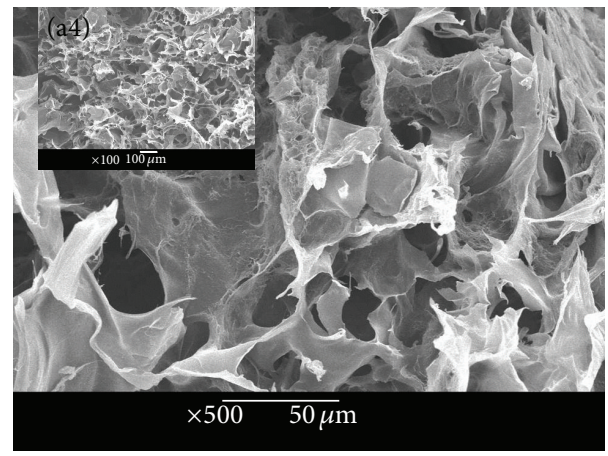

(b4)

Figure 1: SEM micrographs of the surface sections of PVA hydrogel and PVA with additives: ((a1), (b1)) IPN-1 with 15\% w/v PVA; ((a2), (b2)) IPN-2 with 2\% w/v gelatin; ((a3), (b3)) IPN-3 with 5\% w/v gelatin; ((a4), (b4)) IPN-4 with 7\% w/v gelatin; after 1 and 3 of freezing-thawing cycles, respectively. Scale bar (a1)-(a4): $100(\mu \mathrm{m}) ;(\mathrm{b} 1)-(\mathrm{b} 4): 50(\mu \mathrm{m})$. 
with an elastic modulus of $(0.80 \pm 1.50 \mathrm{MPa})$. The IPN hydrogels containing $7 \% \mathrm{w} / \mathrm{v}$ gelatin display the highest tensile strength compared to the other samples. Table 2 shows a tensile strength of $(0.75 \pm 0.18 \mathrm{MPa})$ and the elastic modulus is $(0.98 \pm 2.45 \mathrm{MPa})$. The increment of elastic modulus and tensile strength is attributed to an increase in rigidity, because the IPN hydrogels a hard inorganic component. The extraordinary asset value of strength at break for IPN hydrogels is undoubtedly due to the presence of gelatin, which shows higher gel fraction and more entangled structure in comparison with pure gel. To examine the correlation between mechanical property changes and IPN hydrogels, constant strain-rate compression tests were performed on the IPN hydrogel in order to determine their elastic moduli. Representative stress-strain curves of the IPN hydrogels are presented in Tables 2 and 3 . These results displayed the ability of gelatin concentration and number of cycles to individually affect the compressive modulus of hydrogels. Based on the mechanical property results for our biocomposite IPN hydrogels which are able to attain high tensile strength, it is concluded that there is an opportunity for applications as potential candidate for scaffolds tissue engineering.

3.6. Cytotoxicity of Photocrosslinked IPN PVA/GE Hydrogels. The MTT assay, which is a rapid, standardized, sensitive, and inexpensive method to determine cell viability and proliferation or whether a material contains significant quantities of biologically harmful extracts, is the first step used to screen the biocompatibility of a biomaterials. The effect of PVA, GE, uncross PVA/GE, and cross-linked PVA/GE IPN hydrogels on viability (cytotoxicity) and proliferation of L929 cells was examined using the MTT assay, which is a colorimetric assay that measures the metabolic activity of viable cells. The cytotoxicities of L929 cells on the PVA, GE, uncross PVA/GE, and cross-linked PVA/GE are shown in Figure 2. Based on cytotoxicity results, a fairly acceptable viability of the cells was observed on PVA ( $>88 \%$ at $100 \%$ extract solution), uncross PVA/GE (>94\% at $100 \%$ extract solution), and cross-linked PVA/GE ( $>98 \%$ at $100 \%$ extract solution), while GE membrane showed a slightly higher viability (109\% at $100 \%$ extract solution). Although the biocompatibility of GE was high, the general survival rate of the cells for photocrosslinked PVA/GE scaffold was satisfactory; therefore, the IPN photocrosslinked PVA/GE scaffolds were not toxic to the L929 cells and could be used for tissue engineering scaffolds.

\subsection{Proliferation of Fibroblast Cells L 929 on IPN PVA/GE} Blend. The proliferation of fibroblasts on PVA, GE, uncross PVA/GE, and cross-linked IPN PVA/GE blend was evaluated using MTT assay after 1,3 , and 5 days of incubation as shown in Figure 3. A significantly higher cell proliferation (day 5) in GE and PVA/GE blend compared to PVA was exhibited in Figure 3. After 1 day, the number of cells had exceeded the number of cells initially seeded $\left(10^{4}\right.$ cells/well). As clearly shown in Figure 3, the number of fibroblasts that grew was greater than the number of cells initially seeded even after only 3 days in culture. From 1 day to 5 days in culture, the L 929 cells continually proliferated on including the crosslinked IPN PVA/GE.

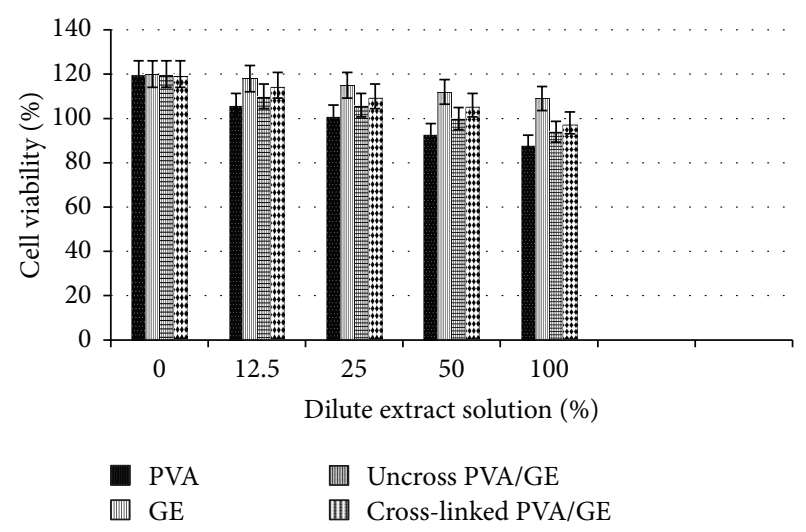

FIgUre 2: Cell viability of PVA, GE, uncross PVA/GE, and crosslinked PVA/GE IPN blend. The MTT assay was used to measure the viability of L929 cells at various dilute extract solution concentrations. Media at $100 \%$ were used as a control. Standard errors were expressed as bar diagram.

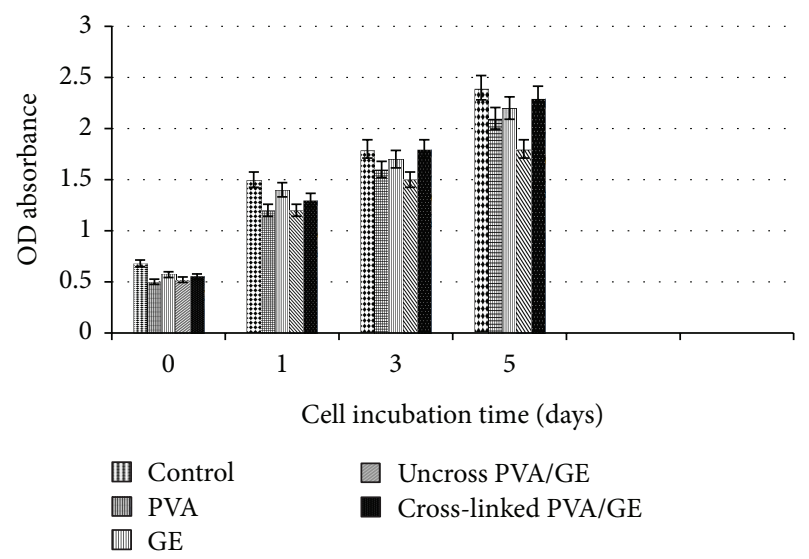

FIGURE 3: Fibroblast cellular proliferation of PVA, GE, uncross PVA/GE, and cross-linked IPN PVA/GE blend after 3 cycles and 1, 3 , and 5 days using the MTT assay. Standard errors were expressed in the bar diagram. Media at $100 \%$ were used as a control and optical density (OD) corresponded to the cell survivability after 1,3 , and 5 days.

Cellular viability assay by MTT was used to find out the percentage of viable cell numbers per extract dilutions after certain periods. However, to observe the increased cell numbers and their real time morphologies, light microscopic studies were conducted. Observation of the morphological changes of cells is a very important means for predicting postcellular responses: spreading, proliferation, and survival. An ideal scaffold must provide the contact guidance for controlling cell adhesion and directing cell migration that influences cell proliferation [36,37]. Figure 4 shows the light microscopic images of cells treated with extract solutions for day 5. For the morphologies' fibroblasts at day 1 , only a little amount of cells was observed. However, the amount of cell numbers was found to increase gradually at day 5 for all samples. Especially, at day 5, cellular growth was almost confluent. However, in case of cross-linked PVA/GE composed of cells, growth pattern was better than that of the 


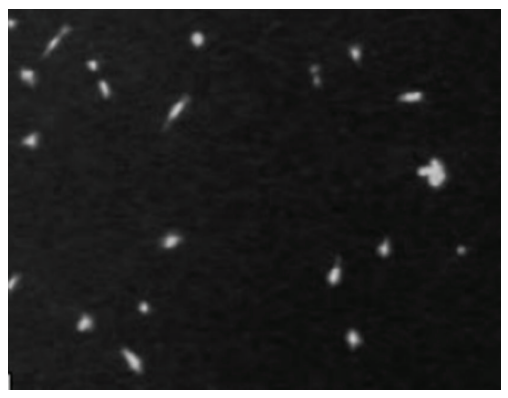

(a)

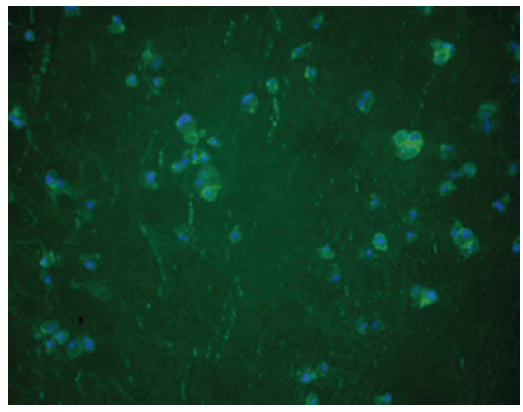

(d)

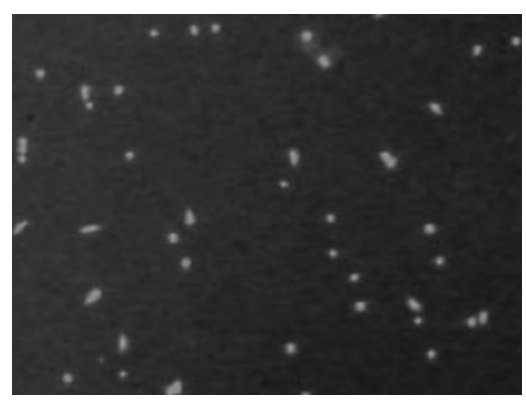

(b)

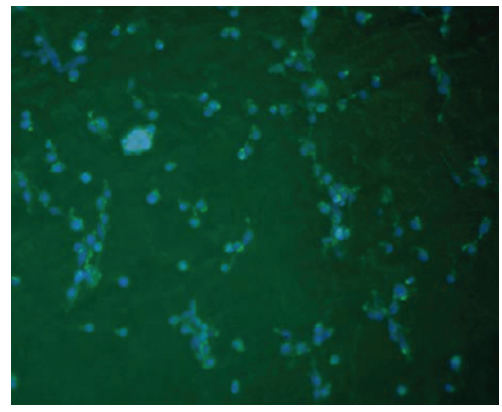

(e)

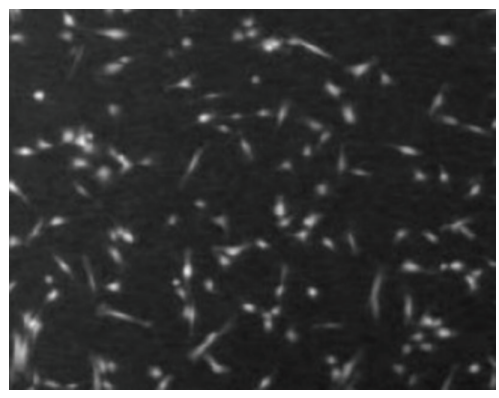

(c)

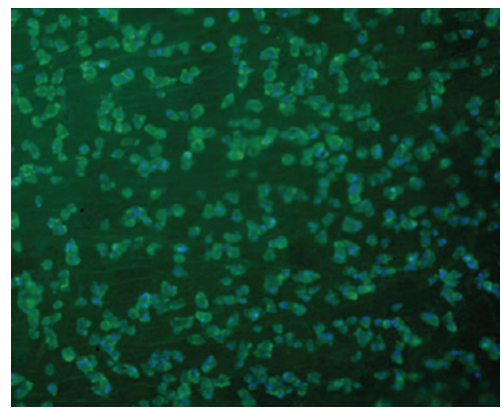

(f)

FIGURE 4: Fibroblast cellular proliferation of uncross PVA/GE and cross-linked IPN PVA/GE blend after 1 ((a), (d)), 3 ((b), (e)), and 5 ((c), (f)) days using the optical microscope observation.

uncrossing PVA/GE. In fact, this result corresponds with the cell viability and proliferation results very well.

\section{Conclusion}

PVA/GE hydrogels with IPN structure were prepared successfully by enzymatic and cyclic freezing-thawing method. The size and arrangement of these pores are the result of number of freezing-thawing cycles and the presence of crosslinking agents. The IPN PVA/GE hydrogels have the highest values of mechanical properties as evaluated from compression tests, also the cross-linking density showed the highest values. The SEM micrographs of these hydrogels show that the majority of the pores is opened and irregular. These interactions become stronger with increasing number of freezingthawing cycles and results in more regular internal structure. The quantity of gelatin was the key factor to obtain IPN PVA/GE hydrogels with desirable properties. The IPN PVA/GE hydrogels showed excellent physical and mechanical properties, which met the essential requirements for ideal medical applications. Based on swelling measurements, they exhibited high capability in absorbing fluid, so recommended for exudative wounds. In addition, fibroblasts that grew over the cells treated with extract solutions exhibited the appropriate morphology and displayed good proliferation; this indicates that the cross-linked network structure of obtained gels is stable enough, suggesting that developer scaffolds might be used for tissue engineering applications.

\section{Acknowledgment}

This paper is supported by the Project of the National Natural Science Foundation of China (51073036).

\section{References}

[1] J.-H. Juang, S. Bonner-Weir, Y. Ogawa, J. P. Vacanti, and G. C. Weir, "Outcome of subcutaneous islet transplantation improved by polymer device," Transplantation, vol. 61, no. 11, pp. 15571561, 1996.

[2] D.-H. Chen, J.-C. Leu, and T.-C. Huang, "Transport and hydrolysis of urea in a reactor-separator combining an anion-exchange membrane and immobilized urease," Journal of Chemical Technology and Biotechnology, vol. 61, no. 4, pp. 351-357, 1994.

[3] S. H. Hyon, W. I. Cha, Y. Ikada, M. Kita, Y. Ogura, and Y. Honda, "Poly(vinyl alcohol) hydrogels as soft contact lens material," Journal of Biomaterials Science, vol. 5, no. 5, pp. 397-406, 1994.

[4] J. K. Li, N. Wang, and X. S. Wu, "Poly(vinyl alcohol) nanoparticles prepared by freezing-thawing process for protein/peptide drug delivery," Journal of Controlled Release, vol. 56, no. 1-3, pp. 117-126, 1998.

[5] M. T. Razzak, D. Darwis, Z. Zainuddin, and S. Sukirno, "Irradiation of polyvinyl alcohol and polyvinyl pyrrolidone blended hydrogel for wound dressing," Radiation Physics and Chemistry, vol. 62, no. 1, pp. 107-113, 2001.

[6] F. Yoshii, Y. Zhanshan, K. Isobe, K. Shinozaki, and K. Makuuchi, "Electron beam crosslinked PEO and PEO/PVA hydrogels for wound dressing," Radiation Physics and Chemistry, vol. 55, no. 2, pp. 133-138, 1999.

[7] F. Yoshii, K. Makuuchi, D. Darwis, T. Iriawan, M. T. Razzak, and J. M. Rosiak, "Heat resistance poly(vinyl alcohol) hydrogel," Radiation Physics and Chemistry, vol. 46, no. 2, pp. 169-174, 1995.

[8] D. A. Ossipov and J. Hilborn, "Poly(vinyl alcohol)-based hydrogels formed by 'click chemistry," Macromolecules, vol. 39, no. 5, pp. 1709-1718, 2006.

[9] H. K. Purss, G. G. Qiao, and D. H. Solomon, "Effect of "glutaraldehyde" functionality on network formation in poly(vinyl 
alcohol) membranes," Journal of Applied Polymer Science, vol. 96, no. 3, pp. 780-792, 2005.

[10] Z. Ajji, "Preparation of poly(vinyl alcohol) hydrogels containing citric or succinic acid using gamma radiation," Radiation Physics and Chemistry, vol. 74, no. 1, pp. 36-41, 2005.

[11] S. Benamer, M. Mahlous, A. Boukrif, B. Mansouri, and S. L. Youcef, "Synthesis and characterisation of hydrogels based on poly(vinyl pyrrolidone)," Nuclear Instruments and Methods in Physics Research B, vol. 248, no. 2, pp. 284-290, 2006.

[12] P. Martens and K. S. Anseth, "Characterization of hydrogels formed from acrylate modified poly(vinyl alcohol) macromers," Polymer, vol. 41, no. 21, pp. 7715-7722, 2000.

[13] N. A. Peppas and N. K. Mongia, "Ultrapure poly(vinyl alcohol) hydrogels with mucoadhesive drug delivery characteristics," European Journal of Pharmaceutics and Biopharmaceutics, vol. 43, no. 1, pp. 51-58, 1997.

[14] T. Hatakeyema, J. Uno, C. Yamada, A. Kishi, and H. Hatakeyama, "Gel-sol transition of poly(vinyl alcohol) hydrogels formed by freezing and thawing," Thermochimica Acta, vol. 431, no. 1-2, pp. 144-148, 2005.

[15] R. Ricciardi, C. Gaillet, G. Ducouret, F. Lafuma, and F. Lauprêtre, "Investigation of the relationships between the chain organization and rheological properties of atactic poly(vinyl alcohol) hydrogels," Polymer, vol. 44, no. 11, pp. 3375-3380, 2003.

[16] T. Koyano, N. Koshizaki, H. Umehara, M. Nagura, and N. Minoura, "Surface states of PVA/chitosan blended hydrogels," Polymer, vol. 41, no. 12, pp. 4461-4465, 2000.

[17] W.-Y. Chuang, T.-H. Young, C.-H. Yao, and W.-Y. Chiu, "Properties of the poly(vinyl alcohol)/chitosan blend and its effect on the culture of fibroblast in vitro," Biomaterials, vol. 20, no. 16, pp. 1479-1487, 1999.

[18] T. Chandy and C. P. Sharma, "Prostaglandin E1-immobilized poly(vinyl alcohol)-blended chitosan membranes. Blood compatibility and permeability properties," Journal of Applied Polymer Science, vol. 44, no. 12, pp. 2145-2156, 1992.

[19] J. M. H. Yang, M. J. Huang, and T. S. Yeh, "Preparation of poly (acrylic acid) modified polyurethane membrane for biomaterial by UV radiation without degassing," Journal of Biomedical Materials Research, vol. 45, pp. 133-139, 1999.

[20] H. S. Koh, T. Yong, C. K. Chan, and S. Ramakrishna, "Enhancement of neurite outgrowth using nano-structured scaffolds coupled with laminin," Biomaterials, vol. 29, no. 26, pp. 35743582, 2008.

[21] H.G.l, “United States Patent," 4055554, 1977.

[22] U. Gerber, U. Jucknischke, S. Putzien, and H.-L. Fuchsbauer, "A rapid and simple method for the purification of transglutaminase from Streptoverticillium mobaraense," Biochemical Journal, vol. 299, no. 3, pp. 825-829, 1994.

[23] K. Yokoyama, N. Nio, and Y. Kikuchi, "Properties and applications of microbial transglutaminase," Applied Microbiology and Biotechnology, vol. 64, no. 4, pp. 447-454, 2004.

[24] C. W. Yung, L. Q. Wu, J. A. Tullman, G. F. Payne, W. E. Bentley, and T. A. Barbari, "Transglutaminase crosslinked gelatin as a tissue engineering scaffold," Journal of Biomedical Materials Research A, vol. 83, no. 4, pp. 1039-1046, 2007.

[25] M. Carmen Go'mez-Guillén, A. Isabel Sarabia, M. Teresa Solas, and P. Montero, "Effect of microbial transglutaminase on the functional properties of megrim (Lepidorhombus boscii) skin gelatin," Journal of the Science of Food and Agriculture, vol. 81, no. 7, pp. 665-673, 2001.
[26] A. P. Rokhade, S. A. Agnihotri, S. A. Patil, N. N. Mallikarjuna, P. V. Kulkarni, and T. M. Aminabhavi, "Semi-interpenetrating polymer network microspheres of gelatin and sodium carboxymethyl cellulose for controlled release of ketorolac tromethamine," Carbohydrate Polymers, vol. 65, no. 3, pp. 243-252, 2006.

[27] A. P. Rokhade, S. A. Patil, and T. M. Aminabhavi, "Synthesis and characterization of semi-interpenetrating polymer network microspheres of acrylamide grafted dextran and chitosan for controlled release of acyclovir," Carbohydrate Polymers, vol. 67, no. 4, pp. 605-613, 2007.

[28] L. H. Sperling, "Interpenetrating polymer networks and related materials," Journal of Polymer Science Macromolecular Reviews, vol. 12, pp. 141-180, 1977.

[29] T. Kurokawa, H. Furukawa, W. Wang, Y. Tanaka, and J. P. Gong, "Formation of a strong hydrogel-porous solid interface via the double-network principle," Acta Biomaterialia, vol. 6, no. 4, pp. 1353-1359, 2010.

[30] J. E. Folk, “[127] Transglutaminase (guinea pig liver)," Methods in Enzymology, vol. 17, pp. 889-894, 1970.

[31] Chinese standards GB/T, 5009. 3-2003, D.o.m.i.f.

[32] M. Frydrych, C. Wan, R. Stengler, K. U. O’Kelly, and B. Chen, "Structure and mechanical properties of gelatin/sepiolite nanocomposite foams," Journal of Materials Chemistry, vol. 21, no. 25, pp. 9103-9111, 2011.

[33] P. R. Hari and K. Sreenivasan, "Preparation of polyvinyl alcohol hydrogel through the selective complexation of amorphous phase," Journal of Applied Polymer Science, vol. 82, no. 1, pp. 143$149,2001$.

[34] G. Mickisch, S. Fajta, G. Keilhauer, E. Schlick, R. Tschada, and P. A lken, "Chemosensitivity testing of primary human renal cell carcinoma by a tetrazolium based microculture assay (MTT)," Urological Research, vol. 18, no. 2, pp. 131-136, 1990.

[35] American National Standard, Biological Evaluation of Medical Devices-Part 5: Tests for Cytotoxicity, 1999.

[36] J. Fukuda, A. Khademhosseini, Y. Yeo et al., "Micromolding of photocrosslinkable chitosan hydrogel for spheroid microarray and co-cultures," Biomaterials, vol. 27, no. 30, pp. 5259-5267, 2006.

[37] A. J. Horobin, K. M. Shakesheff, and D. I. Pritchard, "Promotion of human dermal fibroblast migration, matrix remodelling and modification of fibroblast morphology within a novel $3 D$ model by Lucilia sericata larval secretions," Journal of Investigative Dermatology, vol. 126, pp. 1410-1418, 2006. 

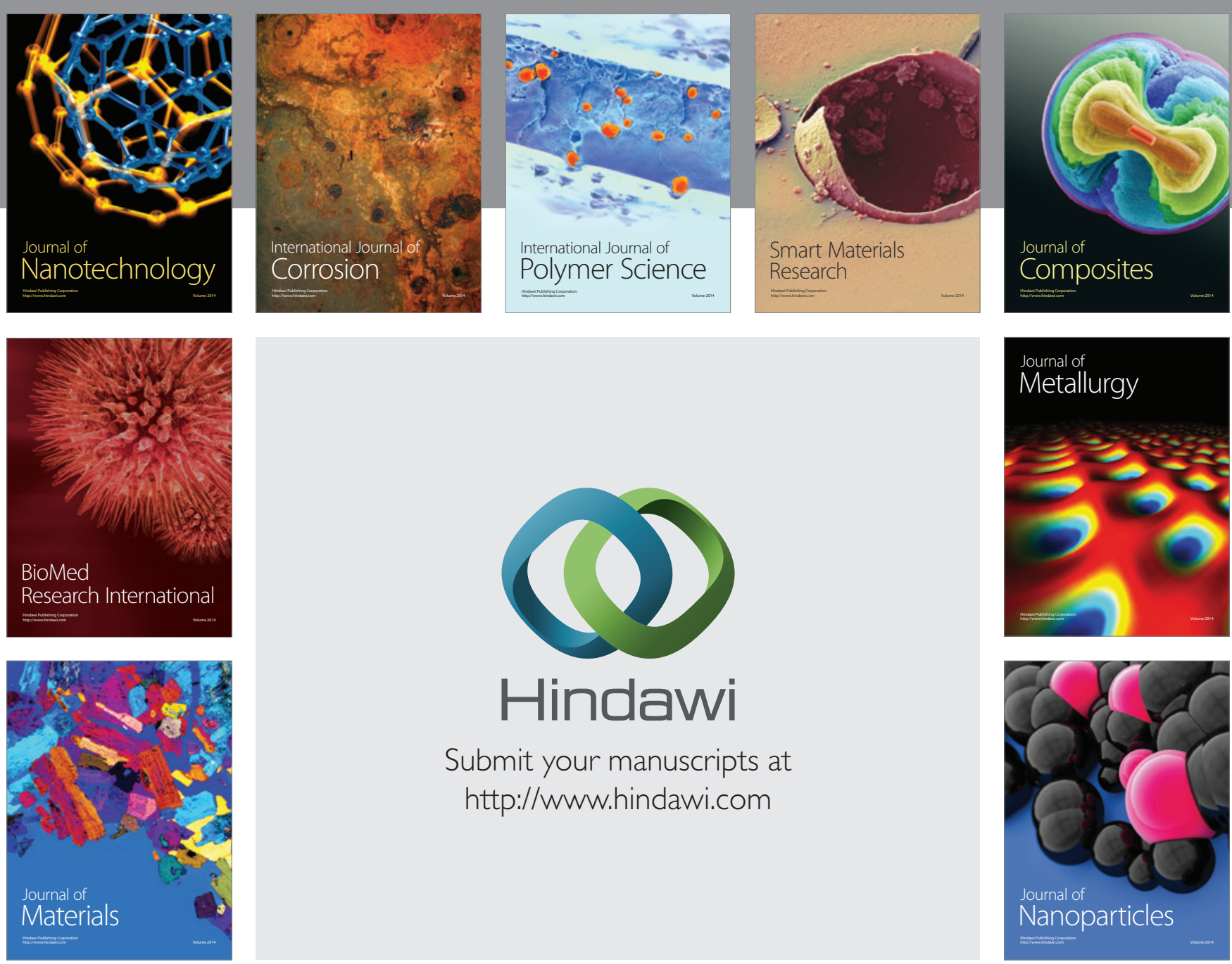

Submit your manuscripts at http://www.hindawi.com
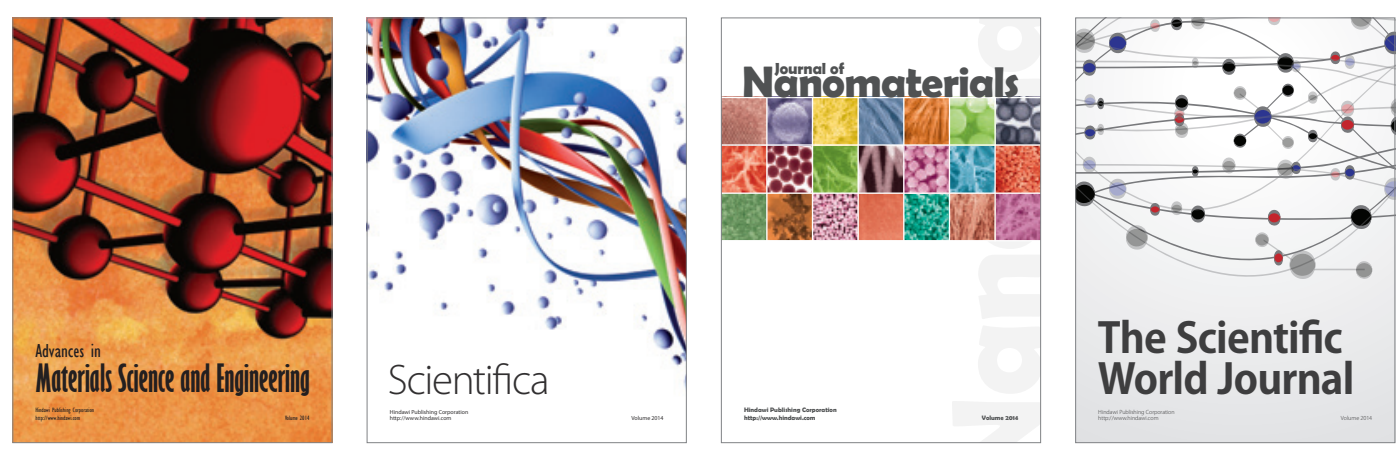

\section{The Scientific World Journal}
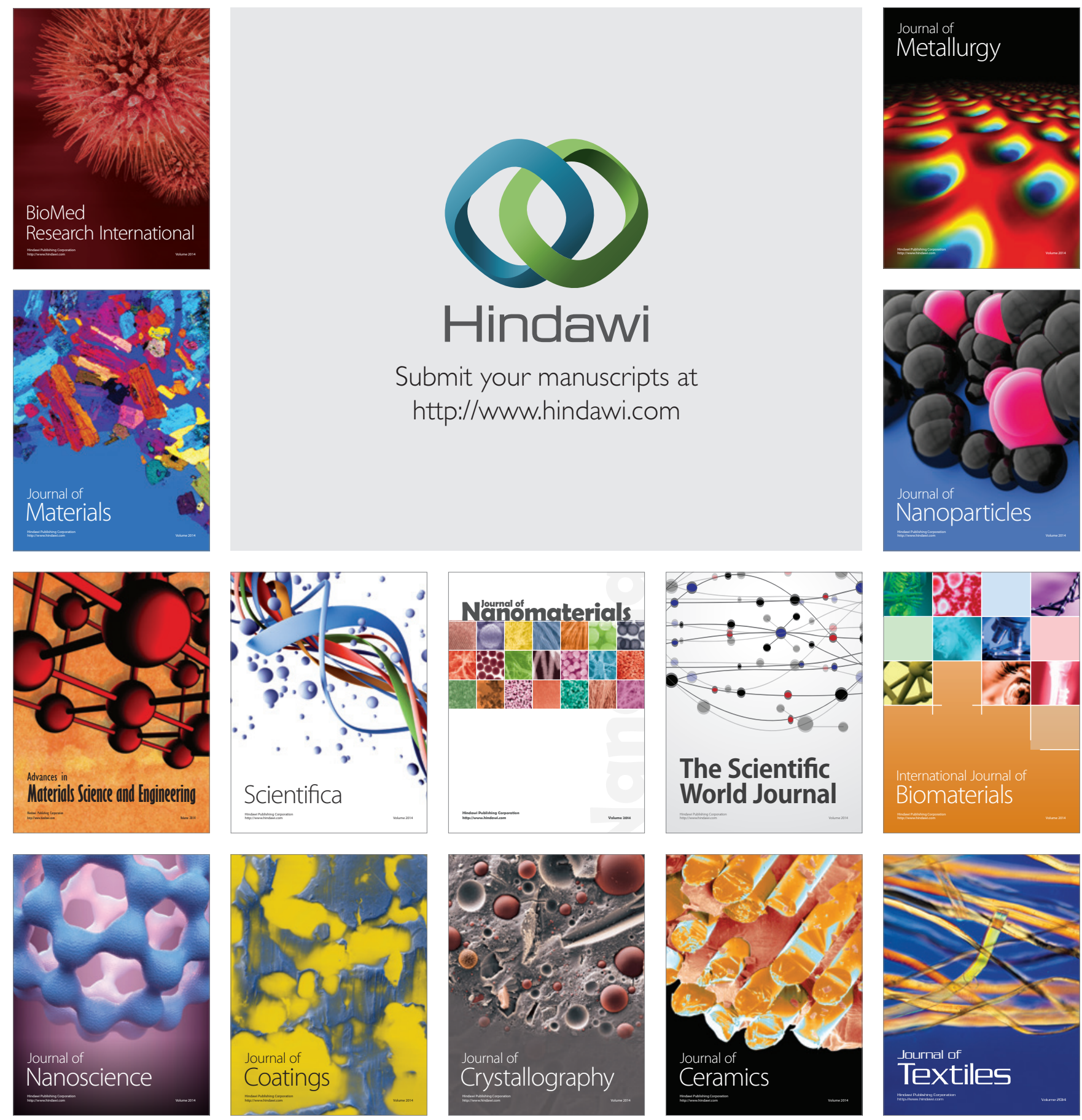